\title{
Ultra short-time dynamics of radiation damage in fec metals
}

\author{
Marc Hayoun ${ }^{1}$, Gerrit Coddens and Guillaume Petite \\ Laboratoire des Solides Irradiés, Ecole Polytechnique, CEA-DSM-IRAMIS, CNRS, 91128 \\ Palaiseau, France
}

\begin{abstract}
We have performed molecular-dynamics simulations of displacement cascades in copper in order to investigate the non-equilibrium ultra-short time damage and to evaluate the possibility of observing it experimentally in-situ (e.g. in a pump/probe laser experiment). The atomic trajectories have been analysed by calculating their X-ray diffraction patterns as a function of time. The results show that an integrated X-ray intensity can indeed be used to evidence the irradiation effects. Even though the number of Frenkel defects is large, the main effect of the irradiation showing up in the X-ray intensities at ultra-short times is an important alteration of the lattice vibrations. On the basis of these results, a new pump/probe set-up is proposed.
\end{abstract}

Keywords: x-ray diffraction; point defects; copper; molecular dynamics

\section{Introduction}

Present-day laser permit to obtain very short and intense laser pulses, which can be used to produce many kinds of short and intense bursts of secondary particles with a broad spectrum of energies. These secondary particles can be e.g. neutrons, electrons, protons or X-rays. Ultra-short Xray pulses are usually produced in the interaction of an intense visible ultra-short laser pulse with a hot and dense plasma, using either solid or gaseous targets. Depending primarily on the target atomic number, the X-ray spectrum can consist of sharp spectral lines (e.g. $\mathrm{K}_{\alpha}$ radiation of light elements [1]), broader unresolved transition arrays or even continuous Brehmsstrahlung radiation [2]. In the most recent experiments, where the laser-plasma interaction occurs in the relativistic regime, synchrotron radiation of the laser-driven electrons can produce high-intensity broadband emission in the 1-10 keV photon energy range, the so-called "betatron radiation" [3]. Likewise, in

\footnotetext{
${ }^{1}$ Email: marc.hayoun@polytechnique.edu
} 
this relativistic regime, femtosecond pulses of high-energy electrons and protons (the latter with energies up to tens of $\mathrm{MeV}$ ) can be obtained [4-5]. Their energy spectrum is usually continuous, though the latest developments using reduced-mass targets show that also monochromatic particle beams can be obtained. A specific feature of all such laser-driven pulsed particle sources is that the high-energy radiation emission is precisely timed with the driving laser pulse, opening the possibility of "pump-probe" experiments using such sources.

When a high-energy proton impinges onto a solid target it produces a displacement cascade. Many Molecular Dynamics (MD) simulations have been performed to study the physics of such displacement cascades in various solids [6-8], but on certain issues the confrontation between theory and experiment has remained rather indirect. In fact, the simulations show that at short times a displacement cascade produces a large number of Frenkel defects $[9,6,8]$, but these large numbers have never been observed experimentally, as the vast majority of these defects only exist for fleeting moments. They recombine very quickly, e.g. within $10 \mathrm{ps}$, such that only a tiny fraction of the initially created pairs survive this self-annealing process by the time one tries to observe them with different equipment. Similarly, the surplus energy the impinging particle puts into the sample, creating the cascade, which is characterized by a set of larger instantaneous local values for $<\mathrm{u}^{2}>$ over a limited spatial extent, gradually diffuses away with time. To have a chance to observe displacement cascades and their Frenkel defects one must therefore find a way to probe a cascade in situ. Ultra-short laser pulses in a pump/probe set-up provide exactly the combination of experimental facilities needed to observe the cascades. The idea is thus to produce both a pulsed proton beam and a pulsed X-ray beam from two derivations of the femtosecond-pulsed laser beam. Let us assume that we are able to produce a pulsed proton beam of more or less well defined energy. We assume also that we are able to determine exactly at which time $t_{0}$ each pulse will reach the solid target. By varying the path length of the laser beam derivation that produces the X-ray pulses one can then choose the delay time $t$ corresponding to the moment in time $t_{0}+t$ at which we probe the cascade. Hence we could probe the time evolution of the cascade and the Frenkel effects over the whole time span during which they pop in and out of existence. 
The present paper reports on a MD simulation of such a difficult type of experiment. We simulate displacement cascades in a copper single crystal as produced by the impact of a proton with a kinetic energy $K_{p}$ of $49.2 \mathrm{keV}$. This value corresponds to a kinetic energy $K_{C u}$ of $3 \mathrm{keV}$ for the recoiling copper atom in a head-on proton collision, as calculated from the relation [10]:

$$
K_{C u}=4 K_{p} \frac{M_{p} M_{C u}}{\left(M_{p}+M_{C u}\right)^{2}} \cos ^{2} \psi,
$$

where the angle $\psi=0$ in a head-on collision; $M_{p}$ and $M_{C u}$ are the masses of the incident and target particles, respectively. Multiple recoils within a single cascade, corresponding to the impinging proton hitting two atoms in the lattice in succession have not been considered. Also possible interactions between two displacement cascades that could be produced more or less simultaneously in an extremely high proton flux have not been considered. The momentum of the proton has been assumed to be along the $y$ direction, which corresponds to the $(010)$ direction of the copper single crystal. Simultaneously we have calculated the X-ray diffraction diagram of the simulation box at different times $t_{0}+t$ after the simulated proton impact at time $t_{0}$. Hence we have monitored the time evolution of this diffraction diagram while the cascade develops. This combination of direct-space and reciprocal-space data should in principle allow us to learn how the diffraction spectra have to be interpreted. We anticipate that this could be a valuable aid for experimental-protocol design, and give added value to the experimental data. In order to avoid confusion, it should be noted that the time dependence of the elastic intensity is not a dynamical signal. In order to obtain a dynamical signal one must correlate the Fourier amplitudes at two different times.

\section{Simulation and analysis of the atomic trajectories}

\subsection{Molecular Dynamics}

The MD $[11-12,6,8]$ computations have been performed in the micro-canonical ensemble. The modelling of the copper interactions relies on an empirical n-body potential of the tight-binding type that reproduces satisfactorily its physical properties [13-15]. The simulated system is a cubic box of size $L^{3}(L=14)$ containing 10976 copper atoms. Periodic boundary conditions (PBC) are employed and the time step is equal to $510^{-16} \mathrm{~s}$. We did not introduce any damping of the motion of the 
boundary atoms [16]. In fact, we did not want that the tool used to regulate the number of artefacts that might occur in our simulations (due to the PBC), could introduce itself some additional perturbation. We therefore have limited our means of control to varying the box size. Kinetic disturbances may thus re-enter the MD box unless its size is large enough and the temperature rises due to the kinetic energy dissipated by the primary knock-on atom (PKA). In order to avoid finitesize effects in the calculations of the X-ray intensities, we have considered the MD box as embedded into a large perfect cubic geometrical crystal (see $\S 2.2$ ).

Twenty-one independent equilibrium configurations of the copper crystal (de-correlated by a time evolution of $10 \mathrm{ps}$ ) have been generated at a temperature of $302 \mathrm{~K}$ and a pressure close to zero for a lattice parameter of $3.6358 \AA$. The zero of the time scale of the twenty-one ensuing irradiation simulations corresponds to these equilibrium configurations. The displacement cascades are initiated at $t=0.005 \mathrm{ps}$ by the transfer of kinetic energy to a single copper atom. This simulates a recoil. We have not introduced statistical distributions for the initial recoil parameters of the PKA. In all twenty-one simulation runs the momentum transfer has always been aligned along the (010) direction of the FFC lattice and the kinetic energy transfer has always been given the same constant value. This unusual choice of initial conditions [6] permits to reduce the complexity of the analysis of the X-ray diffraction patterns by only studying the (010) direction. This is justified in a preliminary study wherein the only aim is to explore the experimental possibilities to observe the ultra-short-time damage in-situ. The statistical distribution of the PKA recoil parameters will be taken into account in a future work including the experiment.

As the number of atoms in the simulation box is small there is a complete loss of any structure in the system if the knock-on energy is taken too large. The energy transferred to the copper atom in our simulations has therefore been fixed at the value of $3 \mathrm{keV}$ in order to avoid such problems.

\subsection{X-ray intensity calculations}

The X-ray intensities in the present paper are expressed in physical units. This means that the wave vector $\boldsymbol{q}$ is expressed by the formula $q=2 \pi / \lambda$, as opposed to the expression $q=1 / \lambda$ often used in crystallography, where traditionally units $2 \pi$ are being used. Here $2 \theta$ is the scattering angle, 
and $\lambda$ the wavelength of the X-rays. We note the transfer of wave vector occurring in the scattering process as $\boldsymbol{Q}=\boldsymbol{q}_{\text {out }}-\boldsymbol{q}_{\text {in }}$. We have adopted a special approach for the calculation of the X-ray intensities $I$. It is well known that they are given by $I=|\mathscr{F}(\boldsymbol{Q})|^{2}$, where $\mathscr{F}(\boldsymbol{Q})$ is the Fourier amplitude. The approach permits to avoid truncation problems and to obtain results that are rigorously exact.

For the calculation of the Fourier amplitude $\mathscr{F}(\boldsymbol{Q})$ of a one-dimensional periodic lattice of $N$ atoms along e.g. the $x$-axis, we restrict ourselves to a set $S$ of $\boldsymbol{Q}$-values $Q=2 \pi k / N a$, where $a$ is the lattice parameter, and $k$ an integer. The crystal lattice points are situated in $x_{j}=j a$, where $j$ is an integer. We obtain then in a straightforward manner [17]:

$$
\mathscr{F}(Q)=\sum_{j=1}^{N} e^{i Q x_{j}}=N \sum_{n} \delta\left(Q-Q_{B}\right)
$$

where $Q_{B}=n(2 \pi / a)$ and $n$ runs over $\mathrm{Z}$. This means that there is a reciprocal lattice of Bragg peaks with weight $N$ in $Q_{B}$. For the remaining $Q$-values in the set $S$, the Fourier amplitude $\mathscr{F}(Q)$ is zero, exactly as would be the case in an infinite perfect crystal in all points of reciprocal space that do not correspond to the location of a Bragg peak. But for the $Q$-values that do not belong to the restricted set $S$ of multiples of $2 \pi / N a$, the algebra no longer reproduces the correct zero result that would apply for an infinite perfect crystal. This is hardly surprising, as we have not calculated the Fourier transform of an infinite crystal. In fact, the algebra is exact for a finite crystal. Our problem is rather that we want to remove the finite-size effects from our calculations. The algebra shows that we can achieve this by making our calculations on the set $S$. This result is easily generalized to three dimensions.

This calculation illustrates why Fast Fourier Transform (FFT) programs cannot be used without observing a number of caveats. They can e.g. give wrong answers for the diffraction spectrum of a periodic lattice if the period of the FFT is not adapted to the lattice period of the crystal, such that the points where the Fourier transform is calculated are not on the set $S$. The FFT is then subject to finite-size truncation problems. One must therefore be careful about interpreting the significance of tails in Bragg peaks in a spectrum obtained from a FFT. Also a number of numerical values for the 
dimensions of the simulation box have to be excluded in order to avoid malfunctioning of the FFT. Given the rather small dimensions of our simulation boxes this would have been a severe limitation.

We have therefore adopted the different approach described above. We consider the cubic simulation box $B$ of size $L^{3}$ as embedded into a large, perfect cubic geometrical crystalline lattice $G$ of size $D^{3}$. That is, we mentally cut a cubic hole $H$ of dimensions $L^{3}$ into the geometrical crystal with exactly the same size $L^{3}$ as the simulation box, and again mentally, enter the simulation box into this hole. This has to be done carefully such that one does not introduce any discontinuity in the average inter-atomic distances across the boundaries between $G \backslash H$ and B. E.g., in order to avoid lattice parameter mismatch problems that could be induced by the variation of the lattice parameter with temperature, the simulations have been carried out at constant volume. The Fourier transform to be calculated is then obtained as $\mathscr{F}[G]-\mathscr{F}[H]+\mathscr{F}[B]$. Both $\mathscr{F}[G]$ and $\mathscr{F}[H]$ can be calculated analytically, and the rigorous result can be coded directly into the program. The ensuing results are exact: E.g. the Bragg peaks contained in the Fourier transform $\mathscr{F}[G]$, and calculated according to equation (2), are pure Dirac measures without any tails. The simulation box $B$ is sufficiently small to allow for a calculation of its Fourier transform $\mathscr{F}[B]$ that is purely based on its definition. Hence we avoid this way the truncation effects inherent to the FFT mentioned above. Actually, we perform these calculations on the atomic positions. A real crystal is the convolution of its set of atomic positions $(G \backslash H) \cup B$ with an atomic decoration. In our case this atomic decoration consists of copper atoms. In the Fourier transform, this means that we must multiply the Fourier transform of this set with the atomic form factor of the copper atom. The calculation of this atomic form factor has been performed using the method and the tabular values given in reference [18]. By varying the size $D^{3}$ of the large crystal, we can actually simulate the volume fraction of the crystal that is irradiated in the real experiment. We may note that the copper lattice is not a Bravais lattice and that this leads to extinction rules for some Bragg peaks, which we have calculated. In fact, the FCC unit cell contains four copper atoms and the lattice parameter that has been used is $3.6358 \AA$.

One effect of using PBC is rather important for the calculations of the Fourier transform. The energy and momentum we confer to the PKA are conserved quantities. Eventually they become redistributed over all the atoms of the simulation box. This results in a uniform motion in the $y$ - 
direction of the centre of mass of the simulated lattice. As the energy conferred to this single atom is large on the scale of the lattice dynamics and as the number of atoms in the simulation box is rather limited, this effect becomes readily observable. One by one the lattice planes reach the "upper" boundary of the box in the $y$ direction. There they "jump" - from the viewpoint of the calculation of the Fourier transform - to the "lower" boundary in the $y$ direction, due to the PBC. The phase factor in the Fourier transform that corresponds to this translational "jump" interferes with the phase factor of the Fourier transform of the perfect crystal by the time we square the Fourier amplitudes in order to obtain the X-ray intensities. This introduces a severe error into the calculated intensities under the form of oscillations in the time dependence, that do not correspond to any physical reality. This uniform centre-of-mass motion has therefore been corrected for in the analysis of the computed data.

\subsection{Integration of $X$-ray intensities}

Since the intensities are weak we have integrated them along the line at $q_{z}=0.2 \AA^{-1}$ and $q_{x}=0 \AA^{-1}$. The choice of $q_{z}$ permits to avoid that the strong intensity of the Bragg peaks outweighs the intensities of the small signal we are interested in. The intensities are stored as a function of $q_{y}$ for each selected time of the MD trajectory. The integration is then performed along the $q_{y}$-axis.

\subsection{Frenkel defects}

An atomic site is defined by considering a spherical volume centred on a geometrical regular lattice site. The value of the radius of the sphere is chosen in such a way that a moving atom leaving a regular site is discriminated from a vibrating atom with a large magnitude at the equilibrium temperature. When this volume is empty there is a vacancy on the site. If an atom does not belong to any atomic site it is an interstitial atom. Such a pair of an interstitial atom and a vacancy defines a Frenkel defect. The actual value of the radius of the sphere in the simulations was $0.84 \AA$ This value

can be compared to the vibration amplitude $\left\langle u^{2}\right\rangle^{1 / 2}=0.118 \AA$ at the equilibrium temperature finally reached by the irradiated system, which is $600 \mathrm{~K}$. We have developed a code, based on this definition of the Frenkel defects, to count them in direct space along the MD trajectories.

\subsection{Further analysis}


The code permits to remove a posteriori the Frenkel defects from a given configuration by putting the interstitials on an empty lattice site. The off-Bragg Fourier intensity of the resulting manipulated configuration yields the contribution of the phonons to the X-ray signal. The same code permits also to "kill" a posteriori the phonon dynamics of all on-site atoms in a configuration by putting them on the geometrical lattice position of the site they are "on" in the configuration. The off-Bragg Fourier intensity of the resulting manipulated configuration yields the contribution of the Frenkel defects to the X-ray signal.

\section{Results and discussion}

\subsection{X-ray intensities}

Fig. 1a shows the X-ray diffraction intensities in the $q_{z}=0$ plane obtained from the MD simulation of the $600 \mathrm{~K}$ non-irradiated system. The Bragg peaks can be clearly distinguished. Their intensities are modulated by the Debye-Waller factor. On this intensity scale the thermal diffuse scattering due to phonons or lattice defects is not visible. Therefore, we show on Fig. 1b the intensities from the same simulation in the nearby reciprocal lattice plane $q_{z}=0.2 \AA^{-1}$ where the absence of Bragg peaks permits to visualize the lattice vibrations on a full scale.

These data can be directly compared with those of Fig. 1c. This Fig. displays the X-ray diffraction intensities in the same reciprocal lattice plane $q_{z}=0.2 \AA^{-1}$ from a snapshot at $0.60 \mathrm{ps}$ after knock-on in the MD simulation of the $300 \mathrm{~K}$ irradiated system. We must stress that the time scale of 0.60 ps selected here is much shorter than in traditional simulations. The intensity scales of Fig. $1 \mathrm{~b}$ and $1 \mathrm{c}$ are identical. From the comparison it is obvious that there is additional diffuse scattering intensity in the irradiated system.

This significant extra intensity is the X-ray diffraction fingerprint of irradiation effects in a metal and as such could be used to monitor them. However, in a real experiment the lattice vibrations from the non-irradiated part of the crystal will be superimposed on them. In order to increase the statistics, we have integrated the signal in this plane with respect to $q_{y}$ along the line $q_{x}=0$. All the results presented hereafter will refer to this particular type of integrated intensities. 


\subsection{Integrated $X$-ray intensities}

The result from these integrations as a function of the simulation time is reported in Fig. 2. As mentioned above, the zero of the time scale corresponds to an equilibrium configuration while the knock-on takes place at $t=0.005$ ps. All the data are averages over 21 independent simulation runs. The equilibrium temperature eventually reached by the irradiated system is close to $600 \mathrm{~K}$. We have therefore also performed 21 equilibrium MD simulations on a non-irradiated system for comparison. The associated integrated X-ray intensities are also reported in Fig. 2.

It is obvious that the time-dependence of the intensity in the non-irradiated system is featureless and that the intensities are much lower than those of the oscillating signal from the irradiated system. Hence, this oscillating time dependence is characteristic of an irradiated sample.

\subsection{Rôle of theFrenkel defects}

In order to understand the origin of this large oscillating signal we have identified the positions of all vacancies and interstitials (the off-site atoms), i.e. of all Frenkel pairs. After this, we have repositioned artificially in each configuration all other atoms on geometrical lattice sites. This enabled us to calculate the contribution of the Frenkel defects to the oscillating signal. This contribution is also displayed in Fig. 2 and it can be seen to be very small. Only up to 1 ps does it represent some observable intensity. The contribution from the on-site atoms was obtained alternatively by repositioning artificially all off-site atoms on the open lattice sites. It coincides almost perfectly with the total integrated X-ray intensity.

In summary we i) conclude that the contribution of the Frenkel defects can be ignored, and ii) we attribute the oscillating signal therefore in terms of lattice vibrations that have been altered by the developing cascade.

Fig. 3 validates further the analysis of the contribution from the Frenkel defects. It compares the X-ray intensity due to the Frenkel defects discussed above with the number of these defects obtained by direct counting along the MD trajectory. In fact, both quantities display exactly the 
same time dependence. The small second peak at $1.8 \mathrm{ps}$ is an artefact of the $\mathrm{PBC}$, as will be discussed in section 3.5.

\subsection{Long-time scale}

Fig. 4 displays the behaviour of the time-dependence of the integrated X-ray intensities on a much longer time scale and with a coarser time step than in Fig.2, for two single MD runs. Up to $15 \mathrm{ps}$ the signal from the irradiated sample is significantly larger than the signal from the $600 \mathrm{~K}$ non-irradiated system. On the long-time scale however, the intensities of the two signals become comparable, in agreement with our statement that the equilibrium temperature reached by the irradiated system is approximately $600 \mathrm{~K}$.

\subsection{Size effects}

We remind the reader that we have made sure that there are no finite-size effects in the calculation of the Fourier transforms (see $\S 2.2$ ).

In order to estimate the importance of finite-size effects in our MD simulations, we have performed calculations for two additional sizes of the simulations box, viz. one with $8788(L=13)$ and one with 13500 atoms $(L=15)$. In Fig. 5 we show the same signal as in Fig. 2 for the three box sizes. The signal is clearly size-dependent.

In Fig. 6 we show the same results after a renormalization of the time scales with the linear dimension of the simulation box. On this effective time scale the size dependence no longer shows up. We interpret this result as evidence that the X-ray intensities result mainly from acoustic phonons. This also means that the size effects in our MD simulations are well understood and that they do not bias our conclusions.

The interpretation in terms of acoustic phonons is justified by the following argument. In text books [17] phonons are introduced by solving the problem of a linear chain of $N$ identical atoms of equal masses $m$, equidistantly positioned at equilibrium sites $x_{j}=(j-1) a$, where $a$ is the interatomic distance. $L=N a$ is then the total length of the chain. In the simplest approach only first-neighbour interactions are considered and the force of these interactions is introduced in terms 
of identical springs with a force constant $\kappa$. A set of coupled linear differential equations is then derived for the displacement $u_{j}$ of each atom $j$ from its equilibrium position $x_{j}$ :

$$
m \frac{d^{2} u_{j}}{d t^{2}}=\kappa\left(u_{j-1}-2 u_{j}+u_{j+1}\right),
$$

The solution is a Bloch wave of the type:

$$
u_{j}=u_{1} \exp \left(i \frac{2 \pi}{N a}(k-1) x_{j}\right) \exp (i \omega t)
$$

where $k$ is an integer in $[1, N]$. This leads to the dispersion curve:

$$
\omega\left(q_{k}\right)=2 \sqrt{\frac{\kappa}{m}} \sin \left(a q_{k} / 2\right),
$$

where $q_{k}=\frac{2 \pi}{N a}(k-1)$ is the modulus of the phonon wave vector. The important point is that the $N q_{k}$-values belong to the discrete set $S$ we introduced in $\S 2.2$. One member of this set $S$ is $q_{1}=0$. The smallest positive member is $q_{2}=\frac{2 \pi}{N a}$. For a finite crystal, the dispersion curve is therefore not a continuous line. A continuous line is only obtained in the limit of an infinite crystal. The dispersion curve rather consists of $N$ discrete points $\left(q_{k}, \omega\left(q_{k}\right)\right)$, where $q_{k}$ belongs to $S$. Hence we have only a discrete set of frequencies in the simulation box, rather than a continuum of frequencies as for an infinite crystal. The smallest non-zero frequency in the box is $\omega\left(q_{2}\right)$. When we change the number of atoms in the simulation box this will modify the value of $\omega\left(q_{2}\right)$ and also all other frequencies that are explored in the box. To render the simulations comparable we must therefore rescale the frequencies. The smallest non-zero frequency is to a very good approximation given by $\omega\left(q_{2}\right)=v q_{2}=v \frac{2 \pi}{L}$, where $v$ is the speed of sound, such that $\omega\left(q_{2}\right) \propto \frac{1}{L}$. Hence we see that the rescaling can be carried out with the linear size $L$ of the simulation box.

Another size effect is illustrated in Fig. 3. The small second peak at $1.8 \mathrm{ps}$ is an artefact due to the PBC. This is proved by the time-rescaled data shown in the insert. When the size of the MD box is increased the height of this peak decreases. Although this time rescaling is numerically identical to the one described above for the phonons, its physical origin is conceptually different. The time rescaling accounts here for the longer time it takes for the cascade to reach the boundary, assuming a constant velocity of propagation. The decrease of the peak when the MD box size is increased 
indicates that it is an artefact related to the size of the MD box. It is due to a part of the cascade leaving the MD box and re-entering it at the other side due to the PBC.

\section{Summary and conclusions}

In summary, we have carried out a MD simulation of displacement cascades in copper. The atomic trajectories have been studied by conventional direct-space analysis, which shows that the number of Frenkel defects is large. In addition, the trajectories have been characterized by calculating their X-ray diffraction patterns as a function of time. These indicate that even at ultrashort times the main effect of the irradiation is an important perturbation of the lattice vibrations.

An important conclusion of our work is that the ideal protocol for probing the ultra-short time physics of displacement cascades should be dynamical rather than static (as in an X-ray diffraction experiment). The relevant analysis tool should then be the dynamical Van Hove correlation function rather than the static correlation function. It may be noted here that rather than X-rays, we could use laser photons to probe the cascade. This offers supplementary possibilities due to coherence effects. E.g. the intrinsic energy resolution of a laser set-up does in principle not permit to observe a phonon. However, due to coherent processes, phonons have nevertheless been observed [19]. As we have observed in the MD simulations that phonons are strongly altered by a displacement cascade at ultra-short times, measuring the phonons optically might be an interesting experimental approach to in situ observation of a displacement cascade. However, it is hard to see how we could use the weak static structure factor results of our present simulations to interpret the experimental data. 


\section{References}

[1] Rousse A, Audebert P, Geindre JP, Falliès F, Gauthier JC, Mysyrowicz A, Grillon G, Antonetti A. Phys. Rev. E 1994;50:2200.

[2] Forget P, Dorchies F, Kieffer JC, Peyrusse O. Chem. Phys. 2004;299:259.

[3] Albert F, Shah R, Ta Phuoc K, Burgy F, Rousseau JP, Rousse A, Pukhov A, Kiselev S. Int. J. of Mod. Phys. B 2007;21:497.

[4] Malka V, Faure J, Gauduel YA, Lefebvre E, Rousse A, Ta Phuoc K. Nature Physics 2008;4:447.

[5] Borghesi M., Cecchetti CA, Romagnani L, Antici P, Audebert P, Fuchs J, D'Humières E, Brambrink E, Toncian T, Pipahl A, Willi O, Lefebvre E. Int. J. of Mod. Phys. B 2007;21:590.

[6] Bacon DJ. in Computer simulation in materials science, edited by Kirchner HO, Kubin LP, Pontikis V. Dordrecht: Kluwer Academic Publishers; 1996. p. 189.

[7] Bacon DJ, de-la-Rubia T. J Nucl Mater 1994;216:275.

[8] Doan NV, Rossi F. Solid State Phenomena 1993;30-31:75.

[9] Barbu A, Martin G. Solid State Phenomena 1993;30-31:179.

[10] Balanzat E, Bouffard S. Solid State Phenomena 1993;30-31:7.

[11] Allen MP, Tildesley DJ. Computer simulation of liquids. Oxford: Clarendon Press; 1987.

[12] Ciccotti G, Frenkel D, McDonald IR. Simulation of liquids and solids. Amsterdam: NorthHolland Physics Publishing; 1987.

[13] Rey-Losada C, Hayoun M, Pontikis V. Mat Res Soc Symp Proc 1993;291:549.

[14] Ackland GJ, Vitek V. in Atomistic simulation of materials: beyond pair-potentials, edited by Vitek V, Srolovitz DJ. New York: Plenum; 1989. p. 193.

[15] Rosato V, Guillopé M, Legrand B. Phil Mag 1989;A59:321.

[16] Diaz de la Rubia T, Guinan MW. Mater Res. Forum 1992;97-99:23.

[17] Ziman JM. Principles of the theory of solids. New York: Cambridge University Press; 1972.

[18] Waasmaier D, Kirfel A. Acta Cryst 1995;A51:416.

[19] Lindenberg AM, Kang I, Johnson SL, Missalla T, Heimann PA, Chang Z, Larsson J, Bucksbaum PH, Kapteyn HC, Padmore HA, Lee RW, Wark JS, Falcone RW. Phys. Rev. Lett. 2000;84:111. 


\section{Figure captions}

Fig. 1. Three-dimensional reciprocal-space plots of the normalized calculated X-ray intensity $I$ $v s .\left(q_{x}, q_{y}\right)$ for three non-averaged single-history runs. Each tick on the $q_{x}$ and $q_{y}$ axes corresponds to $1 \AA^{-1}$. The temperature of the non-irradiated system was chosen close to the final equilibrium temperature of the irradiated system for comparison. a) Bragg peaks in the plane $q_{z}=0 \AA^{-1}$ for the non-irradiated system at $600 \mathrm{~K}$. b) Intensities in the off-Bragg plane $q_{z}=0.2 \AA^{-1}$ for the same nonirradiated system at $600 \mathrm{~K}$. c) Intensities in the same off-Bragg plane $q_{z}=0.2 \AA^{-1}$ for the irradiated system at $300 \mathrm{~K}$ after a relaxation time of $0.60 \mathrm{ps}$.

Fig. 2. Time evolution of the integrated X-ray intensities (averaged over 21 independent runs) for the system irradiated at $300 \mathrm{~K}$ (full line) and for the non-irradiated system at $600 \mathrm{~K}$ (dotted line). The temperature of the non-irradiated system was chosen close to the final equilibrium temperature of the irradiated system for comparison. A decomposition of the integrated intensities of the irradiated system into two components is also shown: the thick line corresponds to the contribution from the off-site atoms which give rise to the Frenkel defects whereas the full circles represent the complementary intensity from the on-site atoms.

Fig. 3. In the main plot we compare the number of Frenkel defects (full circles) obtained from direct-space counting with the integrated X-ray intensities from the off-site atoms (full line). The latter is the same signal as shown in Fig. 1 but now on an appropriate scale. The arrows identify the scale for each quantity. Both quantities are averages obtained from 21 independent runs. The small second peak at $1.8 \mathrm{ps}$ is an artefact of the PBC. The insert gives the evidence for this. It displays the number of Frenkel defects in this peak for the three different sizes of the MD box used. The time has been rescaled by the linear dimension of the MD box as discussed in the text. The full circles correspond to the 10976-atoms cell as in the main plot, the diamonds to the 8788 -atoms cell and the open circles to the 13500 -atoms cell. 
Fig. 4. Evolution of the integrated X-ray intensities on a long-time scale of single runs for the system irradiated at $300 \mathrm{~K}$ (full line) and for the non-irradiated system at $600 \mathrm{~K}$ (grey line). As i) the curve does not represent an average over independent runs and as ii) its portion between 0 and $10 \mathrm{ps}$ has been drawn with a poorer time resolution than in Fig. 1, the visible intensity variations are not identical to those observed in Fig. 1. One observes that the irradiated system has reached equilibrium after $30 \mathrm{ps}$ and that this equilibrium state is characterized by intensity fluctuations similar to those of the non-irradiated system at $600 \mathrm{~K}$. Hence, the short-time signal is truly characteristic of the irradiation.

Fig. 5. Time evolution of the integrated X-ray intensities (averaged over 21 independent runs) for the three different sizes of the MD box used for the irradiated system at $300 \mathrm{~K}$ : the full line corresponds to the 10976-atoms cell, the full circles to the 8788 -atoms cell and the open circles represent the 13500 -atoms cell. The signal is clearly size dependent.

Fig. 6. The same time evolution of the integrated X-ray intensities as displayed in Fig. 4 after the rescaling of the time axis by the linear dimension of the MD box discussed in the text. This rescaling counterbalances the size dependence of the simulation. 

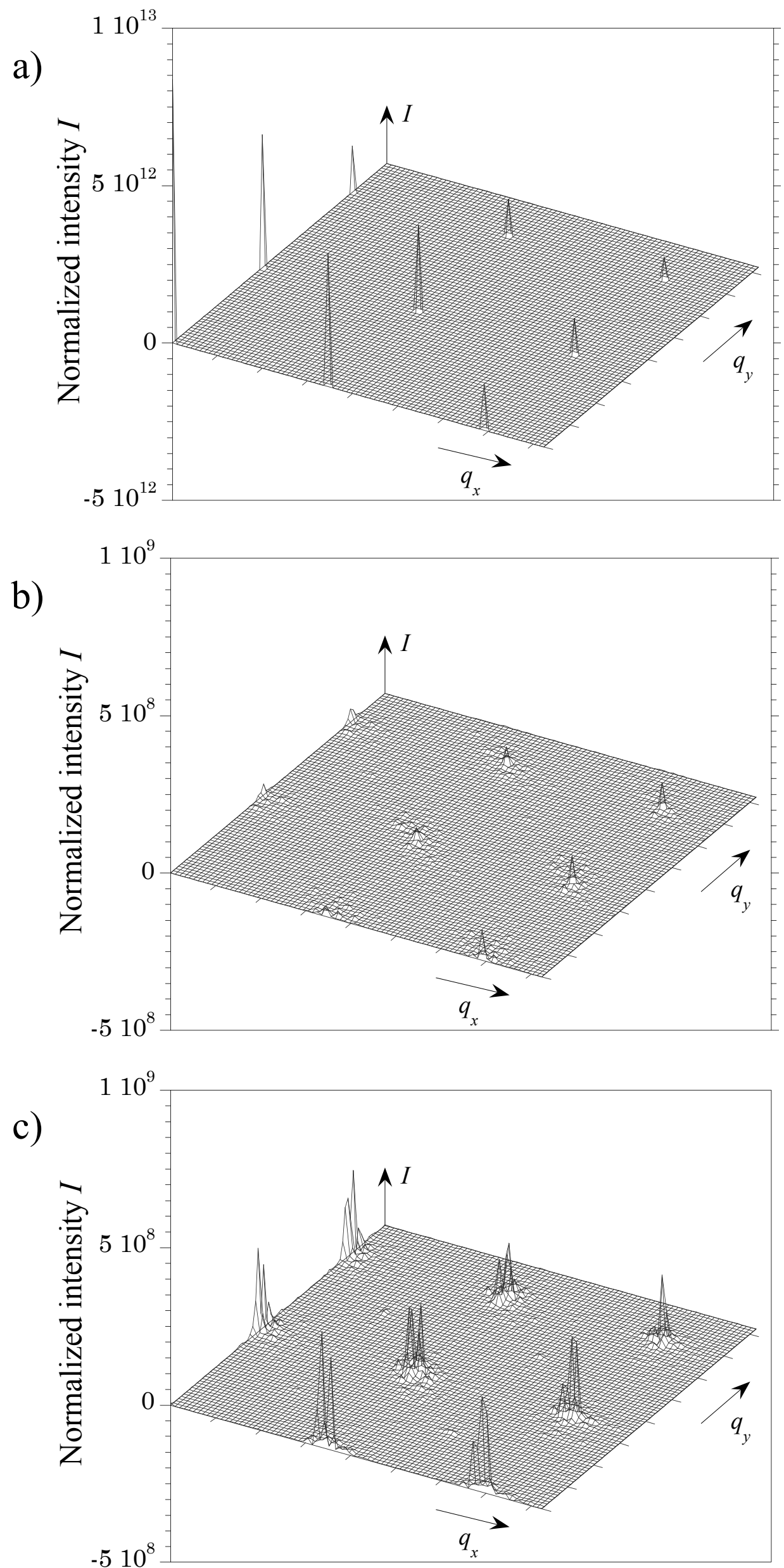


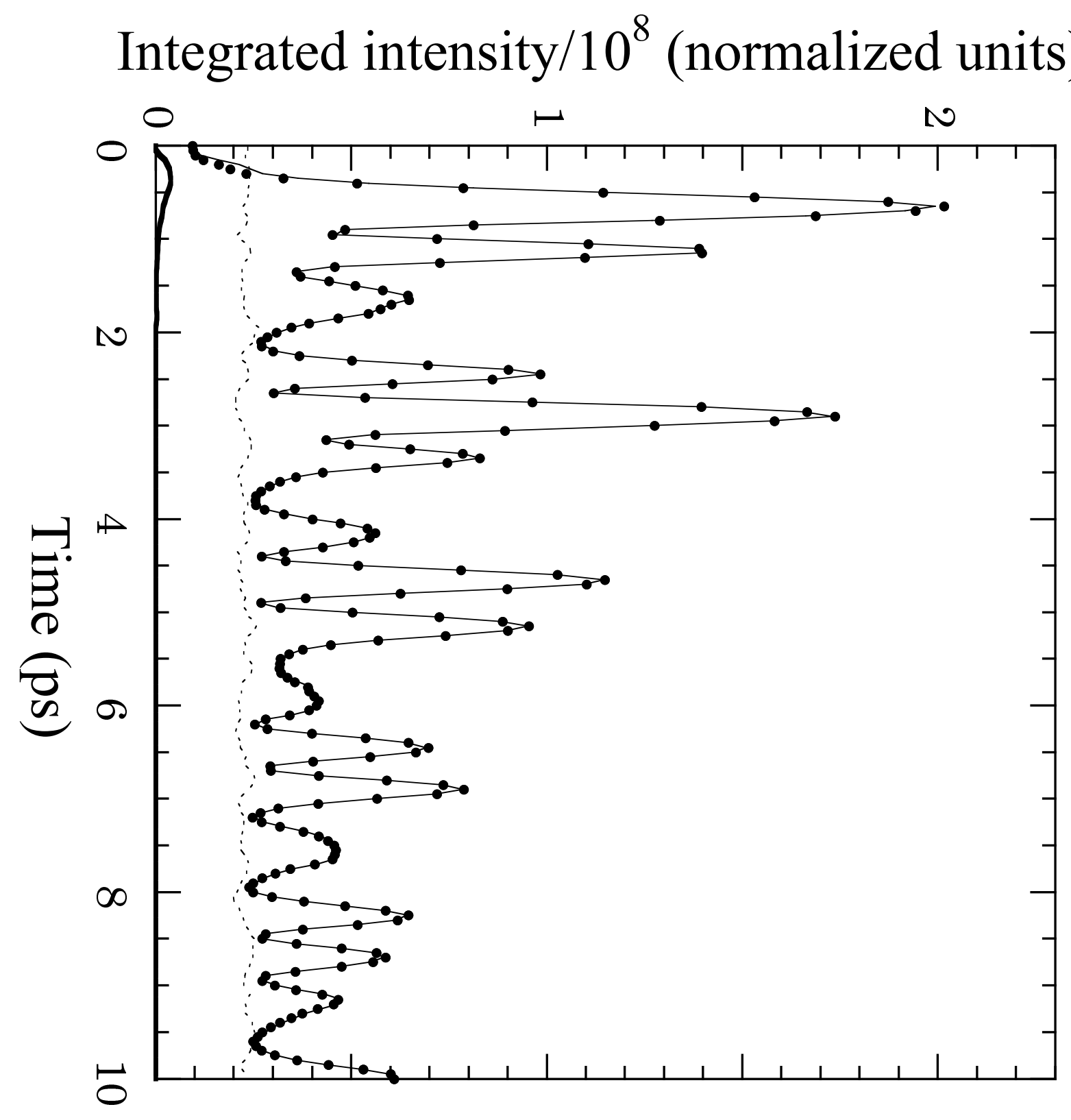




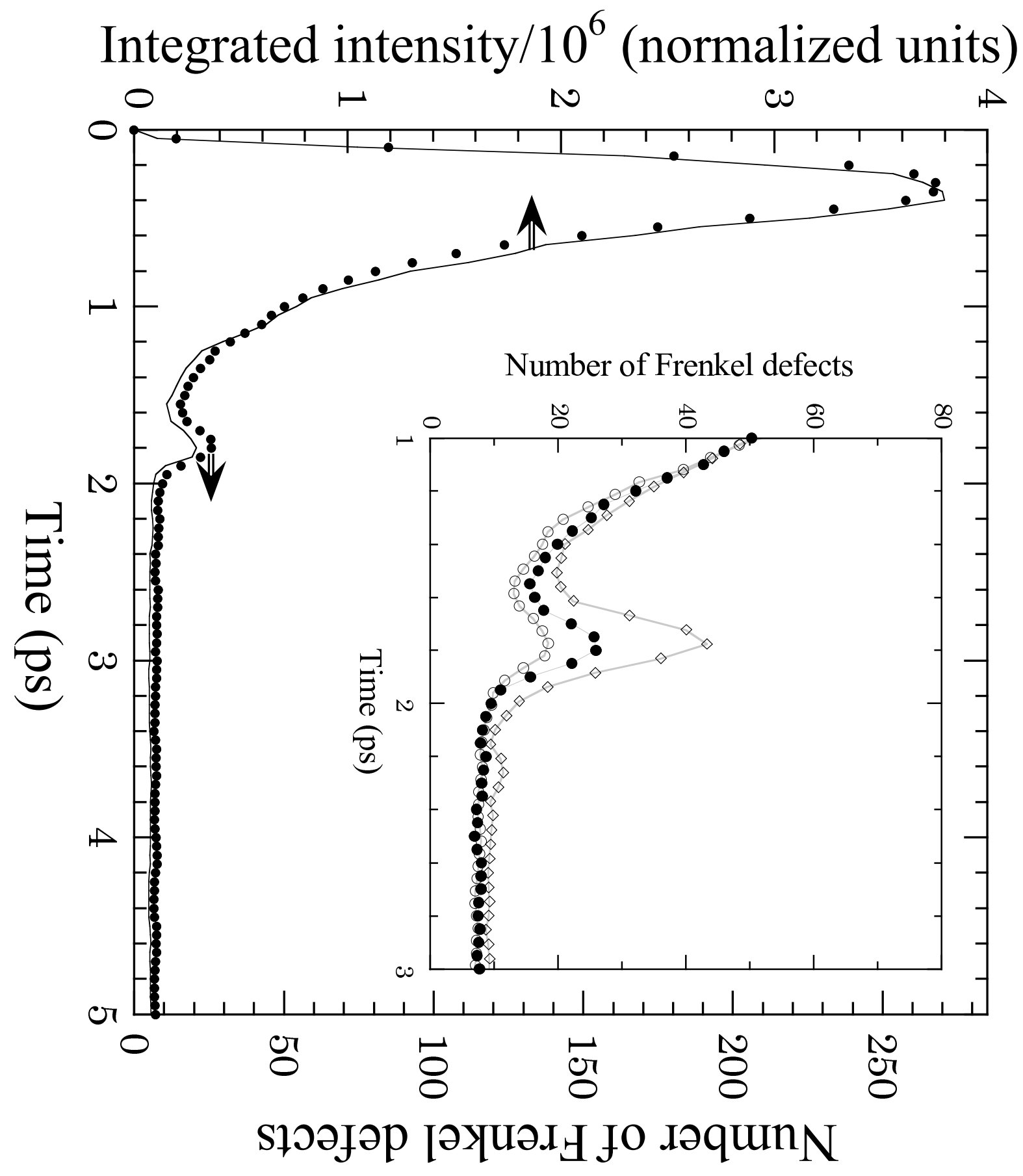




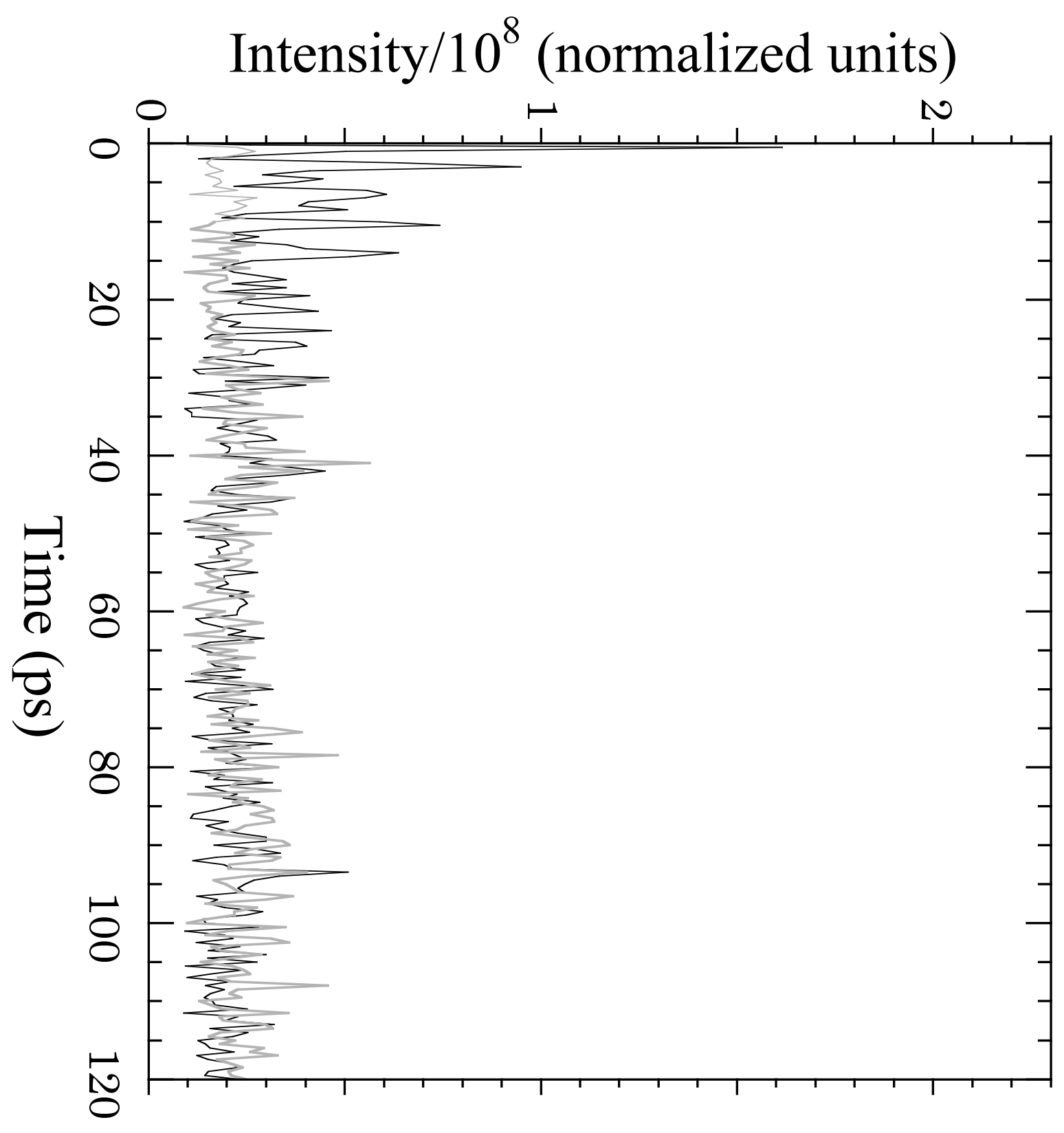


Integrated intensity $/ 10^{8}$ (normalized units)

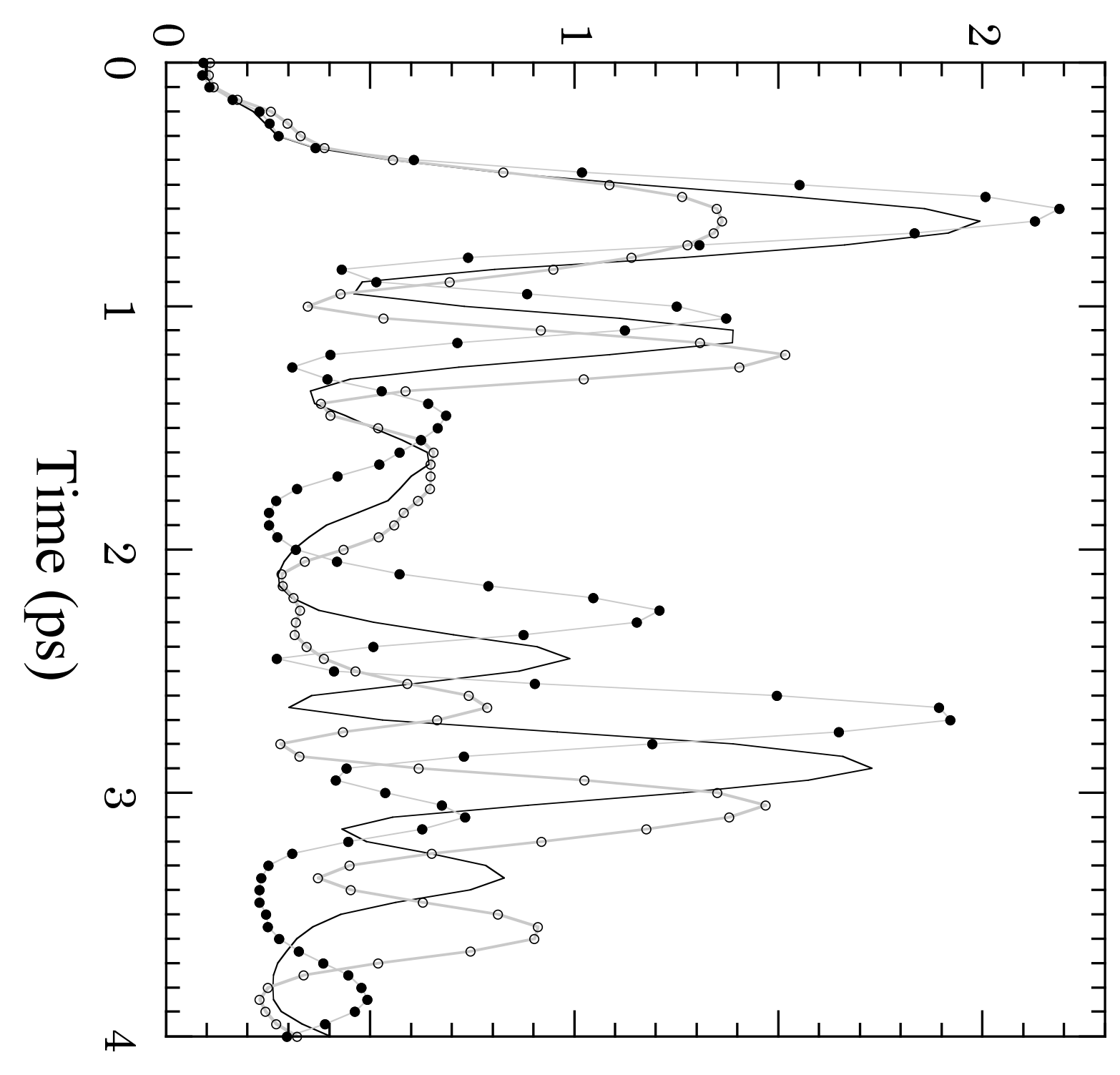




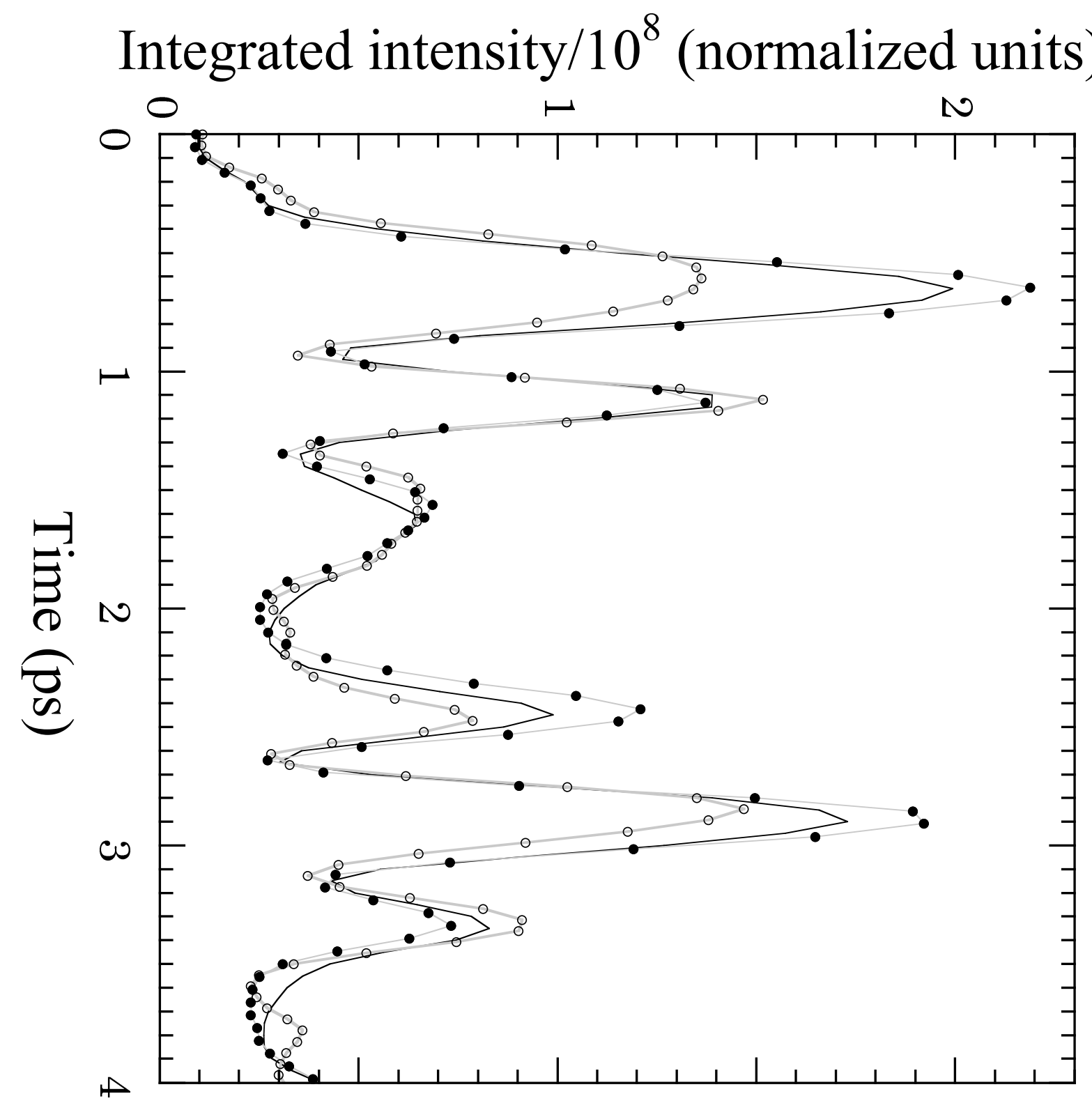

